\title{
Design Team Performance: A CoMParison BeTWEen Self- Formed TEAMS AND TEAMS WiTh Diverse CogNitive MODES
}

\author{
Michele Hastie \\ Department of Process Engineering and Applied Science, Dalhousie University \\ michele.hastie@dal.ca
}

\begin{abstract}
The impact of cognitive mode diversity on team performance and student satisfaction was assessed qualitatively and quantitatively in a capstone chemical engineering design course. In the capstone design course, students were permitted to form their own teams and the distribution of cognitive modes was assessed. In a concurrent design course, the same group of students performed projects in instructor-formed teams that optimized the distribution of cognitive modes. The results indicated no significant difference in team satisfaction between teams that had different levels of cognitive diversity. Although trends seemed to indicate higher rank in the course and greater independence and creativity for groups with higher cognitive diversity, these differences were not statistically significant. Generally, students seemed to have similar experiences in student-formed and instructor-formed groups. However, qualitative comments seem to indicate that groups may have worked more professionally and cohesively in the more cognitivelydiverse, instructor-formed groups.
\end{abstract}

Keywords: capstone design, team formation, cognitive modes, teamology, Myers-Briggs Type Indicator, MBTI

\section{INTRODUCTION}

To maximize creative problem solving, design teams should be a diverse group of students with a broad range of skills, knowledge bases, and backgrounds [1]. There are different kinds of diversity within teams: experiential, professional, sociological, and cognitive. If a team consists of members with different personalities and ways of thinking, each member can bring different approaches when solving problems and making decisions. This can have a positive effect on the overall creativity of the team and quality of their final product.

If an instructor aims to create student teams with diverse cognitive styles, one challenge is that the differences in cognitive modes can be too subtle to easily detect. In response to this challenge, Dr. Douglass Wilde, mechanical engineering professor at Stanford University, developed a twenty-question questionnaire that can be completed by the students prior to group formation [2]. The questionnaire results can help students better understand themselves and their role within the team. Some instructors also observe that, if the questionnaire results are used to form teams, these teams will produce a higher-quality product than teams that are self-selected or formed randomly [2]. This increased performance can be attributed to the increased level of cognitive diversity within the team. Wilde refers to this method of team formation as teamology.

During the final year of the undergraduate chemical engineering program at Dalhousie University, students work in teams of three or four to complete a capstone design course over two semesters. In addition, the same cohort of students complete a chemical engineering process and plant design project, also in teams of three or four. In the 2018-2019 academic year, students were permitted to form their own teams for the capstone project. Once formed, students filled out the questionnaire to determine their preferred cognitive modes. For the plant design course project, the instructor used the results from the questionnaire to form the groups, ensuring that no student was placed in a group with another member from their capstone group.

The performance and teamwork abilities of the groups and information provided by individual student reflections were analyzed to determine if there were any significant differences between groups that had more or less cognitive variety.

\subsection{Cognitive Mode Questionnaire}

The questionnaire developed by Wilde uses the personality theory of Carl Jung to determine a responder's cognitive mode. According to Jung's theory, there are eight cognitive modes, as shown in Fig. 1. Wilde developed a quantitative transformation of the questionnaire results to determine a responder's preference for each of the four mode pairs, depicted by arrows in Fig. 1 .

The questionnaire is similar to the well-known MyersBriggs Type Indicator (MBTI) personality test and uses many of the same concepts. The results identify the responder's preferred methods for information collection (extraverted or introverted and sensing or intuition) and their preferred methods for decision-making (extraverted or introverted and thinking or feeling). 


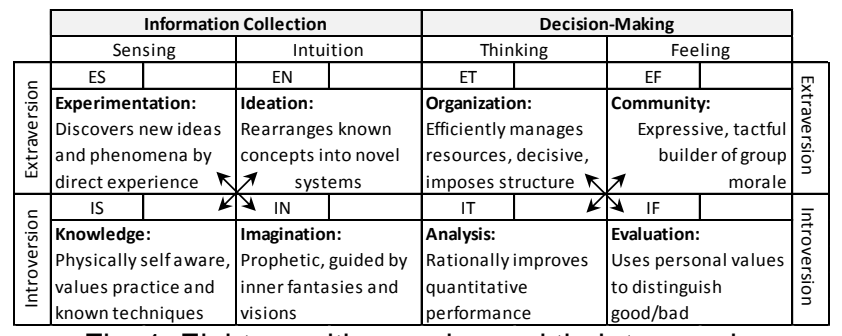

Fig. 1. Eight cognitive modes and their team role descriptions $(\mathrm{E}=$ Extraverted, $\mathrm{I}=$ Introverted, $\mathrm{S}=$ Sensing, $\mathrm{N}=$ iNtuition, $\mathrm{T}=$ Thinking, $\mathrm{F}=$ Feeling) .

It is important to mention that the responses for item $\mathrm{PJ} 3$ of the questionnaire were mistakenly switched in the original publication by Wilde [2]. The corrected version of the questionnaire is shown in Fig. 2 (those who prefer flexibility work better with pressure and those who prefer structure do not). The correct version of the questionnaire is published in a second book by Wilde [3]. The questionnaire is also currently available online, but this version unfortunately has the mistake for PJ3 [4].

\begin{tabular}{|c|c|c|c|}
\hline \multicolumn{4}{|c|}{ Energy Direction: Outward or Inward } \\
\hline $\begin{array}{l}\text { EI1 } \\
\text { EI2 } \\
\text { EI3 } \\
\text { EI4 }\end{array}$ & $\begin{array}{l}\text { You are more: } \\
\text { You are more: } \\
\text { You prefer: } \\
\text { You learn } \\
\text { better by: } \\
\text { You are more: }\end{array}$ & $\begin{array}{l}\text { (e) sociable } \\
\text { (e) expressive } \\
\text { (e) groups } \\
\text { (e) listening } \\
\text { (e) talkative }\end{array}$ & $\begin{array}{l}\text { (i) reserved } \\
\text { (i) contained } \\
\text { (i) individuals } \\
\text { (i) reading } \\
\text { (i) quiet }\end{array}$ \\
\hline \multicolumn{4}{|c|}{ EI difference: $\Sigma \mathrm{e}-\Sigma \mathrm{i}=\mathrm{EI}$} \\
\hline \multicolumn{4}{|c|}{ Orientation: Flexible or Structured } \\
\hline $\begin{array}{l}\text { PJ1 } \\
\text { PJ2 }\end{array}$ & $\begin{array}{l}\text { You are more: } \\
\text { You prefer } \\
\text { activities to be: } \\
\text { You work } \\
\text { better: } \\
\text { You prefer: } \\
\text { You are more: }\end{array}$ & $\begin{array}{l}\text { (p) casual } \\
\text { (p) open-ended } \\
\text { (p) with pressure } \\
\text { (p) variety } \\
\text { (p) improvisational }\end{array}$ & $\begin{array}{l}\text { (j) systematic } \\
\text { (j) planned } \\
\text { (j) without pressure } \\
\text { (j) routine } \\
\text { (j) methodical }\end{array}$ \\
\hline \multirow{2}{*}{\multicolumn{4}{|c|}{$\begin{array}{l}\text { PJ difference: } \Sigma p-\Sigma j=P J \\
\text { Information Collection Process: Facts or Possibilities }\end{array}$}} \\
\hline & & & \\
\hline $\begin{array}{l}\text { SN1 } \\
\text { SN2 } \\
\text { SN3 } \\
\text { SN4 } \\
\text { SN5 } \\
\end{array}$ & $\begin{array}{l}\text { You prefer the: } \\
\text { You prefer: } \\
\text { You are more: } \\
\text { You are more: } \\
\text { You prefer the: }\end{array}$ & $\begin{array}{l}\text { (s) concrete } \\
\text { (s) fact-finding } \\
\text { (s) practical } \\
\text { (s) hands-on } \\
\text { (s) traditional }\end{array}$ & $\begin{array}{l}\text { (n) abstract } \\
\text { (n) speculating } \\
\text { (n) conceptual } \\
\text { (n) theoretical } \\
\text { (n) novel }\end{array}$ \\
\hline \multicolumn{4}{|c|}{ SN difference: $\Sigma \mathrm{s}-\Sigma \mathrm{n}=\mathrm{SN}$} \\
\hline \multicolumn{4}{|c|}{ Decision-Making Process: Objects or People } \\
\hline $\begin{array}{l}\text { TF1 } \\
\text { TF2 } \\
\text { TF3 }\end{array}$ & $\begin{array}{l}\text { You prefer: } \\
\text { You are more: } \\
\text { You see } \\
\text { yourself as } \\
\text { more: } \\
\text { You are more: } \\
\text { You think } \\
\text { judges should } \\
\text { be: }\end{array}$ & $\begin{array}{l}\text { (t) questioning } \\
\text { (t) skeptical } \\
\text { (t) impartial }\end{array}$ & $\begin{array}{l}\text { (f) accommodating } \\
\text { (f) tolerant } \\
\text { (f) merciful }\end{array}$ \\
\hline
\end{tabular}

Fig. 2. Cognitive mode questionnaire [2].

The numerical results for EI, JP, SN, and TF are used to calculate a score for each of the eight cognitive modes (the calculations are described by Wilde [2]). For example, a positive score for ES in Fig. 1 (extroverted sensing) suggests a preference for this cognitive mode. The magnitude of the score indicates the strength of that preference. It would also result in a negative score of the same magnitude for the opposite mode in the pair, in this example, IN (introverted intuition). It is also possible to score 0 for both modes in a pair, suggesting equal preference for both.

Upon completing the questionnaire, a responder will typically receive a result that suggests that they prefer four cognitive modes - two information collection modes (ES or IN and EN or IS) and two decision-making modes (ET or IF and EF or IT). In cases where an individual scores 0 for a pair, their result may show more than four preferred modes.

To maximize team effectiveness, members that strongly prefer certain cognitive modes - for example, "extroverted sensing" - should take on roles within the group that strongly rely on this style of thinking - for this example, "experimentation".

\section{COURSES AND TEAM FORMATION}

The author was the instructor for two final year design courses in the chemical engineering program at Dalhousie University. The details of these courses are summarized in Table 1. Together, the courses had a cohort of 67 students and 56 of them completed both courses in the same year.

Table 1: Descriptions of the courses used in the study.

\begin{tabular}{|l|l|c|}
\hline \multicolumn{1}{|c|}{ Course } & \multicolumn{1}{c|}{ Duration } & $\begin{array}{c}\text { Number of } \\
\text { students }\end{array}$ \\
\hline Capstone design & Fall and winter & 59 \\
\hline Plant design & Winter & 64 \\
\hline
\end{tabular}

\subsection{Capstone Design Course}

In the capstone design course, students were permitted to form their own teams of either 3 or 4 students. This resulted in 15 teams, 11 of which were "student-formed" and 4 were formed by the instructor based on project interests.

After teams were formed, the class completed the cognitive mode questionnaire. The results were then used to determine the team pattern. This allowed the teams to identify their own cognitive mode and how they fit within the team. They could also identify missing cognitive modes within their team. In this situation, Wilde recommends that a student who has a low score in the opposing cognitive mode pair be called upon to be responsible for this missing mode [2]. This is referred to as "reaching for a vacant mode".

\subsection{Plant Design Course}

When it came time to form teams in the winter semester for the other design course, plant design, the instructor used the questionnaire results to form teams such that they all had high levels of cognitive diversity. The method 
described by Wilde in Chapter 3 of his book was used to form the teams [2]. The objectives were

a) that no student is placed in a team with another member from their capstone design team, and

b) that all teams have at least one member with a positive score in each of the eight cognitive modes

Only 13 of the students reported a preference for the EN mode (extraverted intuition). However, because 17 teams were needed, students who had a low score in the opposite mode (IS) were also used to meet this requirement. This resulted in $6 / 17$ of the teams not meeting objective $b$ ) because they had a team member with a negative score for EN. However, this is acceptable because of the ability for team members to reach and fill in for vacant modes.

Objective b) was also not met for another team because the data for one student was unfortunately entered incorrectly and the correction was only made at the conclusion of the course. However, this team still had members with high scores in six of the eight modes and members with low negative scores in the two missing modes.

\section{RESULTS AND DISCUSSION}

\subsection{Myers-Briggs Type Indicator (MBTI) Results}

The questionnaire provides a rough estimate of the responder's MBTI. These results are shown in Fig. 3 for the entire class of 67 students. A few students scored 0 , which implies an equal preference for both types. The results indicate that there were about the same number of students who prefer extraversion and introversion. However, there was a significant preference for sensing over intuition, thinking over feeling, and judging over perceiving. These results are consistent with other published MBTI results for engineering students [5], [6], [7], [8], [9].

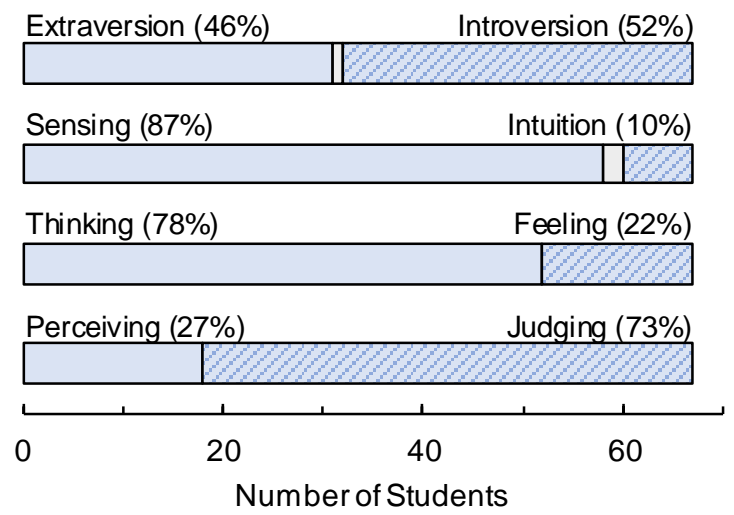

Fig. 3. Distribution of Myers-Briggs Type Indicator (MBTI) results for the class of final year chemical engineering students.

\subsection{Distribution of Cognitive Modes}

In Fig. 4, the frequency (bars) and average score (markers) for each cognitive mode is shown. The four most preferred modes are ES (extraverted sensing / experimentation), IS (introverted sensing / knowledge), ET (extraverted thinking / organization), and IT (introverted thinking / analysis). This is also reflected in the MBTI results in Fig. 3, which indicate an overall class preference for using sensing to collect information and thinking for decision-making.

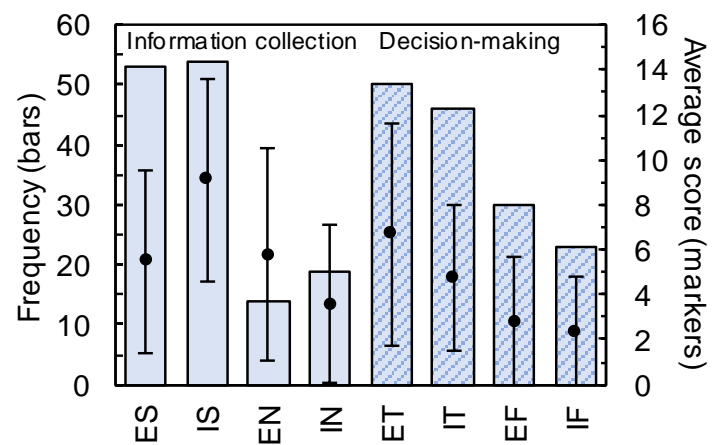

Fig. 4. Frequency (bars) and average score (markers) of each cognitive mode for the class of final year chemical engineering students $(E=$ Extraverted, $I=$ Introverted, $S=$

Sensing, $\mathrm{N}$ = iNtuition, $\mathrm{T}=$ Thinking, $\mathrm{F}=$ Feeling). The errors represent one standard deviation.

The preference of ES and IS suggests that students are more likely to prefer team roles that require knowledge over ideation and experimentation over imagination. This is not surprising considering that most engineering professionals rely on knowledge of observed and understood scientific phenomena. However, this could indicate a lack of students (and future engineers) who have a strong sense of intuition, which is arguably a requirement for creative problem solving [10]. There are three possible reasons for this result:

a) The results are accurate but are not problematic. The inclination of sensing over intuition may be wellaligned with the type of work performed by and skills needed of working engineers.

b) The results are accurate and are potentially problematic. If the workforce is lacking engineers who use intuition to collect information when problem solving, there is a potential need in the design curriculum to further develop this style of thinking and further encourage those who already have this preference.

c) The results are inaccurate. When answering the questionnaire, students might feel biased toward sensing activities because there is an unconscious perception that they are more representative of the expected thinking patterns of an engineer.

It is difficult to conclude which of the above scenarios is the case. However, it would be interesting to determine 
whether this discrepancy in information collection modes has a positive or negative effect on the output of working engineers and if there is a need to address this.

Similar to the sensing attribute, there is an overall preference toward ET (extraverted thinking) and IT (introverted thinking) when making decisions. Again, this is not surprising because engineering work often involves decision-making based on availability of resources and data analysis. However, referring to the descriptions of the feeling modes in Fig. 1, the results show that there could be a lack of graduating students who are comfortable using community and personal values in their decision-making processes, which could be important for decisions that involve communities and other stakeholders.

\subsection{Comparison of Student-Formed and Instructor-Formed Teams}

\subsubsection{Capstone Course (student-formed teams)}

To compare the teams' level of cognitive variety in the capstone design course, the results of each student's questionnaire were used to determine each team's cognitive mode pattern. The total number of team members that reported a preference for a particular mode were added to give a total member count for that mode. Scores of zero were included because they still represent a preference for that mode, but empty scores represent a lack of preference. For an easier comparison between teams, the number of students in each mode were than transformed into a percentage of the total count of modes in the team. An assumption was made that an ideally diverse team would have an equal number of members with a preference for each mode. In other words, each cognitive mode should have a percentage of $1 / 8=12.5 \%$ of the total count. To attain a measure of the disparity from this theoretical ideal, the standard deviation of the percentages was calculated. A low value for standard deviation suggests more cognitive diversity among the team members. The results are presented in Fig. 5. The team numbers are not the same as those used in the course, and only the author is aware of the true identity of the teams.

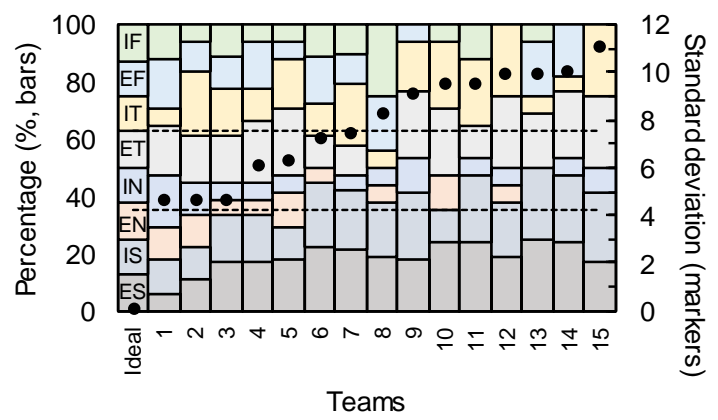

Fig. 5. Distribution of the eight cognitive modes for the capstone design teams. The dashed lines represent the acceptable range of team cognitive diversity. The team numbers are not the same as those used in the course.
The standard deviations ranged from 4.6 to 11 . The team that had the lowest standard deviation, and hence the greatest cognitive diversity, was Team 1 . The team had between one and three group members with a preference for each mode, and none of the modes showed significant dominance over the others.

The team that had the highest standard deviation, and hence the lowest cognitive diversity, was Team 15 . Only five of the eight cognitive modes were represented. In addition, IS (introverted sensing) was the dominant information collection mode and ET and IT (extraverted and introverted thinking) were the dominant decisionmaking modes.

\subsubsection{Plant Design Course (instructor-formed teams)}

In the plant design course, the teams were formed by the instructor based on the principles of teamology. The distribution of cognitive modes for this course's teams is presented in Fig. 6. Again, the team numbers are not the same as those used in the course, and only the author is aware of the true identity of the teams.

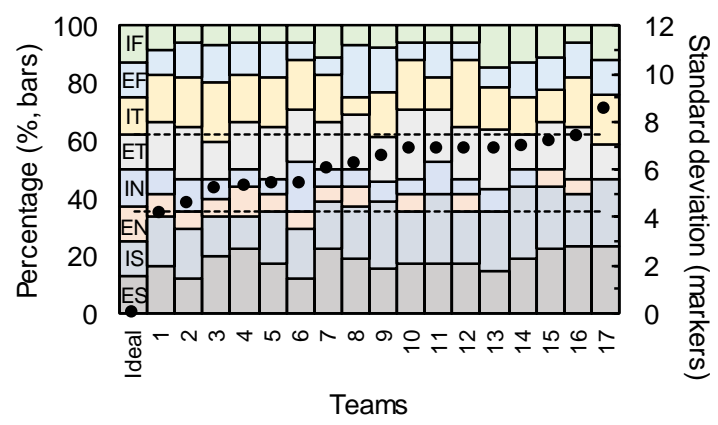

Fig. 6. Distribution of the eight cognitive modes for the plant design course. The dashed lines represent the acceptable range of team cognitive diversity. The team numbers are not the same as those used in the course.

As previously mentioned, one team (Team 15) was formed based on one student's incorrect questionnaire results. As expected, the standard deviation for this team is high since it is missing members with a preference for two of the eight cognitive modes. It is therefore assumed to be an outlier, and that the actual standard deviation ranged between 4.2 and 7.5. These are assumed to be the limits that could be attained by the teamology method for this particular class and is considered to be a range of acceptable levels of cognitive diversity. This range is represented as dashed lines in both Fig. 5 and Fig. 6.

\subsubsection{Comparison between courses}

In the capstone course, five of the teams formed by students and two of the teams formed by the instructor (but not using teamology) were outside the acceptable range. This implies that roughly half (5/11) of the self-assembled teams had a relatively low amount of cognitive diversity within the team compared to the average cognitive 
diversity of the entire class. It is plausible that these teams were formed based mainly on friendships and their mutually similar personality types. In the other half of the teams that had an acceptable level of diversity, randomness may have played a larger role on the cognitive diversity among team members. Even if they considered themselves friends when forming the team, their friendship may have not extended significantly beyond the classroom. These results suggest that when students are allowed to form their own teams, there is roughly a $50 \%$ chance that the team will have a reasonable degree of cognitive diversity.

For the proceeding analysis of the capstone design teams, the teams were separated into three groups (with reference to Fig. 5):

a) High level of cognitive diversity (Teams 1-5)

b) Medium level of cognitive diversity (Teams 6-10)

c) Low level of cognitive diversity (Teams 11-15)

\subsection{Analysis of Team Performance}

The analysis was mainly conducted for the capstone course. This is because the plant design projects were more constrained and structured in comparison to the projects in capstone course. This resulted in less variability between team performance and did not give a good representation of the team's performance in a truly open-ended design project. This course was mainly used to determine the potential range of diversity for the class and as a point of comparison for the students for their reflections at the conclusion of the courses.

The performance of the capstone teams was quantified based on overall team satisfaction, rank in course based on final group average mark, the instructor's evaluation of the group's overall level of independence and creativity, and the number of groups that experienced at least one instance of substantial, non-productive conflict. These results are summarized in Fig. 7. For the first three values, a score of 3 represents outstanding, a score of 2 represents acceptable or average, and a score of 1 represents unacceptable or below average.

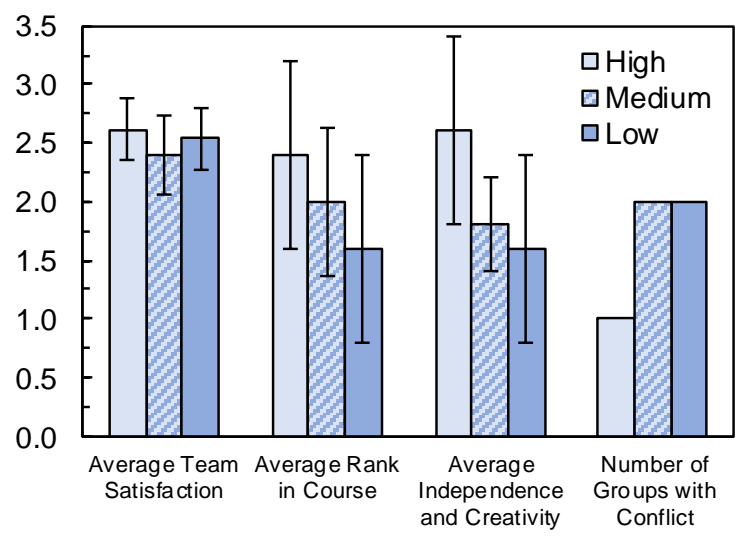

Fig. 7. Team performance for groups with high, medium, and low levels of cognitive diversity. The error bars represent one standard deviation.

\subsubsection{Team Satisfaction}

At the conclusion of the course, each student evaluated their team members' performance with respect to time management, leadership, and overall contribution. It is assumed that the average of these scores is a good representation of that student's overall satisfaction with their team. Each team's average was then used to determine the overall average for those teams with high, medium, and low cognitive diversity, as shown in Fig. 7.

The results did not show any statistically significant differences between the groups for this measurement. This suggests that cognitive diversity did not greatly impact a team's overall team satisfaction with respect to time management, leadership, and overall contribution.

The same measurement was taken in the plant design course, in which teams were formed by the instructor based on teamology. The average team satisfaction was 2.6, which is the same value as the high cognitive variety groups in the capstone course.

\subsubsection{Rank in Course}

The final average team mark was used to rank the teams. They were then divided into three groups: the teams with the top five ranks, the middle five ranks, and the bottom five ranks. These groups were awarded scores of 3, 2, and 1 , respectively.

As seen in Fig. 7, there is a trend that the teams with high levels of cognitive diversity were more likely to receive a final mark that put them in the top third of the class. However, these differences were not found to be statistically significant. Further, all groups (high, medium, and low) had at least one team that was in the top third of the class and one team in the bottom third of the class.

\subsubsection{Independence and Creativity}

At the conclusion of the course, the instructor gave each team a score for their overall level of independence and creativity. The instructor based this score on

a) the overall ability of the group to find a direction in the project, and

b) the overall ability of the group to find creative solutions to problems.

The five teams that most frequently relied on the instructor or the teaching assistants for these two items were given a score of 1 . The five teams that least frequently relied on the instructor or teaching assistants received a score of 3 . The five remaining teams were considered to have an average level of reliance for these two items and were given a score of 2 . It is acknowledged that this score is subjective. However, it did provide a method to determine the effect of a team's cognitive diversity on the creative aspects of the project.

There does appear to be a trend that teams with increasing cognitive diversity were more likely to have an above average level of independence and creativity; however, the difference is not statistically significant. It was noted that $4 / 5$ of the teams with high independence and 
creativity had a high level of cognitive diversity and were not missing any modes. The fifth team in this group of high performers was missing three modes, but was comprised of academically strong students, which may have helped to close this gap.

\subsubsection{Number of Groups with Conflict}

As is expected with student group projects, some teams experienced conflict over the course of the project. The teams with conflicts reported in Fig. 7 were either brought to the attention of the instructor or were described in the reflection submitted by students at the end of the course. These reported conflicts did not include typical "healthy" conflicts that would be expected in a design project (e.g., disagreements over the correct course of action for a design decision). Instead, they were personality conflicts or frustrations over group contribution and commitment.

There was no significantly greater chance for conflict across the different levels of cognitive diversity. However, it is noted that of the five teams with high cognitive diversity, only one team had significant conflict.

Of the five teams that experienced conflict, two were assembled by the instructor and had either high or medium levels of cognitive diversity. Teams with high levels of cognitive diversity can be prone to more conflict. For an individual on this team with a standard set of four cognitive modes, each of these modes will be balanced by another team member with preferred modes on the opposite side of the spectrum for that mode. For example, if a student highly prefers the IS (introverted sensing) mode for knowledge and known techniques, that student may become frustrated with a group member who prefers the EN (extraverted intuition) mode who is often searching for novel ways to implement a design. It is important to help students understand that there will likely be conflict in such cases, but it is for the benefit of the project and should not be taken personally. Because these two teams were formed by the instructor, there may not have been enough familiarity between the students to truly understand this. In both cases, the conflicts were between group members who had very different cognitive mode patterns. In addition, these were situations where one member tried to take on a leadership role in the team whereas the other member resisted this action.

The remaining teams with conflict were all studentassembled and were in teams with medium or low cognitive diversity. One commonality between these teams is that some team members scored very high preferences for certain modes, making it more difficult for them to either work in the opposite mode or value the work being done by a group member in this opposite mode. This could have led to conflict with other group members that scored lower preference values for these modes.

\subsection{Leadership}

Dupont and Hoyle [11] recommend that teams should either have:

a) a single leader with a preference for the EN (extraverted intuition) mode, or

b) two joint leaders each with a preference for IN (introverted intuition) and EF (extraverted feeling), respectively.

While there was not a strong correlation between the leadership score and the EN mode, it was noted that all students with an EN score above 2 were considered leaders within their group.

\subsection{Comments from Reflections}

Students were asked to compare their group experiences at the conclusion of the courses through a written reflection. The majority of students had similar experiences in both groups. Some commented on having a better experience in one course over the other; however, their reasons mostly related to whether or not they had a personal preference for forming their own teams or relying on the instructor to do this.

Other than these comments, a selection of the more relevant reflection comments is provided in Table 2. It is difficult to draw any concrete conclusions from the reflections since it is difficult to determine whether a student preferred a team because of its cognitive diversity, because it happened to have less conflict, or because they enjoyed working with friends. However, some comments stand out.

A few comments mention that there was a lack of familiarity with the plant design team, which either hindered task allocation or was overcome with time. This is common when forming teams for students and highlights the importance of communicating to the class the benefits of having cognitive variety of a team.

One particular student mentioned that they preferred their capstone team because their plant design team required them to be a leader (apparently this student was reluctant to do so). In review of the team's cognitive mode pattern, this particular student was the only member with a score for EN (extraverted intuition) which is a mode recommended for team leaders, so it does make sense that none of the other team members naturally took on a leadership role.

Other comments that could reflect the fact that their plant design team benefited from increased diversity include that they had more support from team members, worked as a team to solve issues, interactions were more professional, everyone enjoyed working on different aspects of the project, and there was an increased sense of collaboration. 
Table 2: Selected comments from student reflections that compared the team experience in both courses.

\begin{tabular}{|c|c|c|}
\hline $\begin{array}{c}\text { Level of } \\
\text { Cognitive } \\
\text { Diversity in } \\
\text { Student's } \\
\text { Capstone Team }\end{array}$ & $\begin{array}{l}\text { Preferred } \\
\text { team }\end{array}$ & $\begin{array}{l}\text { Rationale for preference / other } \\
\text { comments }\end{array}$ \\
\hline Low & Plant design & $\begin{array}{l}\text { friendlier, more rational and } \\
\text { positive }\end{array}$ \\
\hline Low & Plant design & more motivated and enthusiastic \\
\hline Low & Plant design & more productive and motivated \\
\hline Low & Plant design & $\begin{array}{l}\text { better support from team } \\
\text { members }\end{array}$ \\
\hline Low & $\begin{array}{c}\text { Similar } \\
\text { experiences }\end{array}$ & $\begin{array}{l}\text { lack of familiarity with plant } \\
\text { design team made it harder to } \\
\text { assign tasks }\end{array}$ \\
\hline Medium & Capstone & $\begin{array}{l}\text { less likely to take constructive } \\
\text { criticism personally }\end{array}$ \\
\hline Medium & Capstone & $\begin{array}{l}\text { better communication, plant } \\
\text { design group needed me to be a } \\
\text { leader }\end{array}$ \\
\hline Medium & Plant design & $\begin{array}{l}\text { similar communication style and } \\
\text { less conflict }\end{array}$ \\
\hline Medium & Plant design & $\begin{array}{l}\text { took longer to get to know each } \\
\text { other but in the end was more } \\
\text { professional and efficient }\end{array}$ \\
\hline High & Plant design & $\begin{array}{l}\text { interactions were more } \\
\text { professional }\end{array}$ \\
\hline High & Plant design & $\begin{array}{l}\text { better communication, worked as } \\
\text { a team to solve issues }\end{array}$ \\
\hline High & $\begin{array}{c}\text { Similar } \\
\text { experiences }\end{array}$ & $\begin{array}{l}\text { more open communication in } \\
\text { plant design team }\end{array}$ \\
\hline High & $\begin{array}{c}\text { Similar } \\
\text { experiences }\end{array}$ & $\begin{array}{l}\text { in plant design, everyone enjoyed } \\
\text { working on different aspects of } \\
\text { the project }\end{array}$ \\
\hline High & $\begin{array}{c}\text { Similar } \\
\text { experiences }\end{array}$ & $\begin{array}{l}\text { less collaboration in plant design } \\
\text { course because it was not a group } \\
\text { of friends }\end{array}$ \\
\hline High & $\begin{array}{c}\text { Similar } \\
\text { experiences }\end{array}$ & $\begin{array}{l}\text { more collaboration in plant } \\
\text { design team, but less efficient }\end{array}$ \\
\hline High & $\begin{array}{c}\text { Similar } \\
\text { experiences }\end{array}$ & $\begin{array}{l}\text { plant design team was more } \\
\text { efficient and produced higher } \\
\text { quality work }\end{array}$ \\
\hline High & $\begin{array}{c}\text { Similar } \\
\text { experiences }\end{array}$ & $\begin{array}{l}\text { capstone course was more like- } \\
\text { minded }\end{array}$ \\
\hline
\end{tabular}

\section{CONCLUSION}

The cognitive mode questionnaire developed by Douglass Wilde was successfully used to determine the cognitive diversity of teams in the chemical engineering capstone design course. By comparing to a course in which teams were formed to have a high level of cognitive diversity, it was determined that roughly half of the capstone teams self-assembled into cognitively diverse teams, while the remaining teams lacked significant diversity.

The level of cognitive variety did not seem to impact the overall team satisfaction score or the number of significant conflicts that occurred. Teams with a high level of diversity did appear to perform better and have greater creativity and independence; however, this observation was not found to be statistically significant. Anecdotally, the conflicts between students within teams seemed to involve team members with large differences in preferred cognitive modes. In addition, some teams commented that it took time to gain familiarity between team members in the instructor-assembled teams. This stresses the importance of highlighting the benefits of cognitive differences at the beginning of the project. The results of the questionnaire showed that the class had an overall preference toward sensing modes for information collection and thinking modes for decision-making. Future work could involve determining the reason for these preferences and whether the disparities are of concern and require changes in the design curriculum.

Although this study did not reveal any statistically significant results, having cognitive variety on a design team is likely an overall benefit. The wide variety of tasks that need to be completed for a design project rely on different mode preferences, specifically those tasks that involve creativity and ingenuity. It is therefore recommended to use the method described by Wilde to form design teams. The questionnaire is quick and easy for students to fill out and the method for assembling teams, though more time-consuming than forming teams randomly, is well-described in the reference [2]. Even if it is not possible to form teams in this manner, it is still worthwhile to have the teams complete the questionnaire. The results allow students to better understand themselves and their role within a team. Teams can also identify potentially missing modes and identify which groups members might best fill these needed roles.

\section{REFERENCES}

[1] Harvey F. Hoffman, The Engineering Capstone Course: Fundamentals for Students and Instructors. Switzerland: Springer International Publishing, 2014. 144 pp. \{ISBN: 978-3-319-05896-2\}

[2] Douglass J. Wilde, Teamology: The Construction and Organization of Effective Teams. London: Springer-Verlag, 2009. 92 pp. $\{978-1-84800-386-6\}$

[3] Douglass J. Wilde, Jung's Personality Theory Quantified. London: Springer-Verlag, 2011. 116 pp. \{ISBN 978-085729-099-1\}

[4] Douglass J. Wilde, "Cognitive Mode Questionnaire," [Online]. Available: http://rrg.utk.edu/resources/FUTURE _BME/clinical_immersion/Teamology_Survey.pdf. [Accessed 6 May 2019].

[5] Peter Rosati, "Students' psychological type and success in different engineering programs," in Proc. Frontiers in Education Annual Conf. (Pittsburgh, PA; 5-8 November 1997), 4 pp., 1997.

[6] Thomas H. Scott, J. Roger Parsons and J. Elaine Seat, "Use of Myers-Briggs Type Indicator in the University of Tennessee engage Freshman Engineering Program," in ASEE Annual Conf. (Montreal, QC; 16-19 June 2002), 15 pp., 2002. 
[7] Richard M. Felder, Gary N. Felder, and E. Jacquelin Dietz, "The effects of personality type on engineering student performance and attitudes," Journal of Engineering Education, vol. 91, no. 1, pp. 3-17, 2002. Available from https://onlinelibrary.wiley.com/doi/abs/10.1002/j.21689830.2002.tb00667.x

[8] Terrance P. O'Brien, Leonhard E. Bernold, and Duane Akroyd, "Myers-Briggs type indicator and academic achievement in engineering education," Journal of Engineering Education, vol. 14, no. 5, pp. 311-315, 1998. Available from http://citeseerx.ist.psu.edu/viewdoc/summa ry?doi=10.1.1.455.7207

[9] V. Rodríguez Montequín, J. M. Mesa Fernández, J. Villanueva Balsera, and A. García Nieto, "Using MBTI for the success assessment of engineering teams in projectbased learning," International Journal of Technology and
Design Education, vol. 23, no. 4, pp. 1127-1146, 2013. Available from https://link.springer.com/article/10.1007/s1 0798-012-9229-1

[10] Siu-Tsen Shen, Stephen D. Prior, Anthony S. White and Mehmet Karamanoglu, "Using personality type differences to form engineering design teams," Engineering Education, vol. 2, no. 2, pp. 54-66, 2007. Available from https://www.tandfonline.com/doi/abs/10.11120/ened.2007. $\underline{02020054}$

[11] Bryony DuPont and Christopher Hoyle, "Automation and Optimization of Engineering Design Team Selection Considering Personality Types and Course-Specific Constraints," in ASEE Annual Conf. \& Exposition. (Seattle, WA; 14-17 June 2015), 13 pp., 2015. 